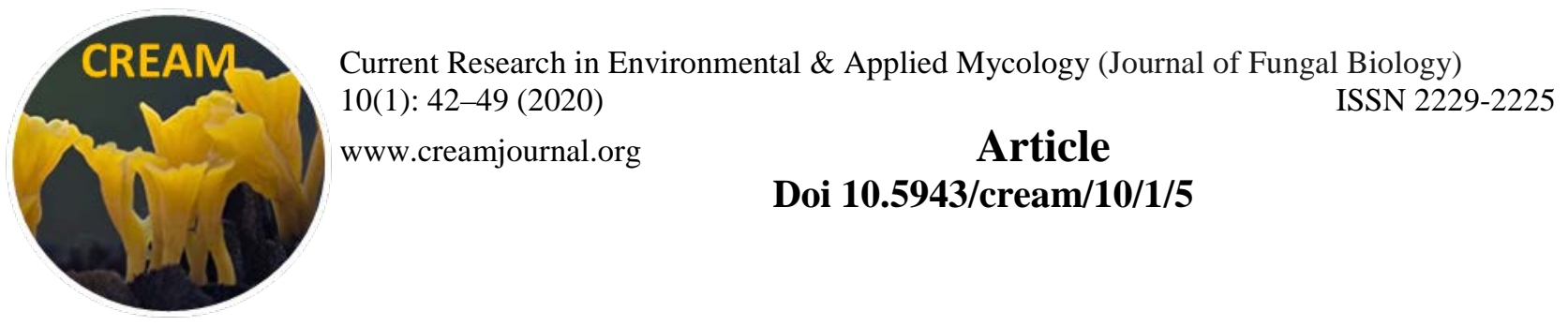

\title{
Annotated checklist of Gymnopilus from Brazil
}

\section{Fabrini FCSS ${ }^{1}$ and Wartchow $\mathrm{F}^{2}$}

\begin{abstract}
${ }^{1}$ Universidade Federal de Pernambuco, Programa de Pós-Graduação em Biologia de Fungos, Av. Nelson Chaves s/n, 50760-420, Recife, PE, BRAZIL

${ }^{2}$ Universidade Federal da Paraíba, Programa de Pós-Graduação em Biologia de Fungos, CEP: 58051-970, João Pessoa, PB, BRAZIL
\end{abstract}

Fabrini FCSS, Wartchow F 2020 - Annotated checklist of Gymnopilus from Brazil. Current Research in Environmental \& Applied Mycology (Journal of Fungal Biology) 10(1), 42-49, Doi 10.5943/cream/10/1/5

\begin{abstract}
A checklist of species belonging to the genus Gymnopilus in Brazil is provided. The list, which includes all species recorded in Brazil prior to 2017, cites 22 species. Brief descriptions, distribution notes, and references are provided.
\end{abstract}

Key words - Agaricales - Agaricomycetes - Neotropic - taxonomy

\section{Introduction}

Historically Gymnopilus P. Karst. has been treated as member of Cortinariaceae by Singer (1986) or Strophariaceae by Kühner (1984) and Guzmán-Dávalos et al. (2003). Recent molecular studies included the genus in Hymenogastrales, but with low support (Matheny et al. 2015). In Brazil, the earliest records of this genus were performed during 19th century with Montagne (1856) and Berkeley \& Cooke (1877). The genus was already treated in previous checklists, on which Putzke (1994) reported 15 and more recently Capelari et al. (2015) 11 species.

In this paper, we present an annotated checklist of Gymnopilus reported from Brazil, providing respective references and data on basidiospores and habitat.

\section{Materials \& Methods}

The checklist was based on a survey of scientific papers and books that cite taxa of Gymnopilus from Brazil. The information retrieved from the literature includes: distribution records per Brazilian state, notes about general habitat and substrates, basidiospore measurements and shapes. For the distribution data, the Brazilian state is listed first followed by the city or other locality information.

Genera and species are listed alphabetically and the authorities for the binomials, as well as the author names spellings, are in according to Robert et al. (2005) and Anonymous (2019) databases.

\section{Results}

Gymnopilus arenicola Hesler

Distribution - São Paulo (Pegler 1997), Mato Grosso do Sul (Bononi et al. 2017).

Habitat - Growing on sandy soil (Pegler 1997). 
Note - According to Hesler (1969), it presents pileus 15-30 mm in diam., smooth or finelly fibrillose, orange to near brownish yellow surface; lamellae adnate to slightly adnexed; veil absent;

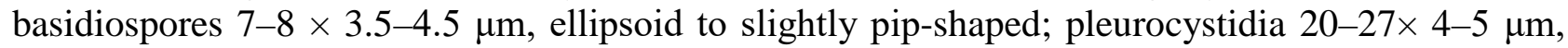
ventricose; cheilocystidia 15-28 × 3-7 $\mu \mathrm{m}$, ventricose to flask-shaped, apex subcapitate or capitate.

\section{Gymnopilus cf. bakeri Dennis}

Distribution - Paraná (De Meijer 2006).

Habitat - On bark of fallen trunk (Dennis 1970).

Note - According Dennis (1970), Gymnopilus bakeri presents pileus 10-20 mm in diam., conic-convex; lamellae adnexed, subdistant, brown; veil none; basidiospores 6-7 $\times 4-5 \mu \mathrm{m}$, ellipsoid, pleurocystidia not mentioned (probably absent); cheilocystidia 25-30 × $6 \mu \mathrm{m}$, apex capitate; clamps not mentioned.

\section{Gymnopilus chrysopellus (Berk. \& M.A. Curtis) Murrill}

Distribution - Rio Grande do Sul (Singer 1953), Santa Catarina (Karstedt \& Stürmer 2008).

Habitat - On dead wood (Hesler 1969).

Note - According Hesler (1969), this species has pileus 25-40 mm, golden yellow, convex then frequently depressed to umbilicate; lamellae adnexed to subdecurrent; veil none; basidiospores 5-7.5 × 3.8-4.7, ellipsoid; pleurocystidia and caulocystidia absent; cheilocystidia 18-27 × 3-6 $\mu \mathrm{m}$, flask-shaped, apex non-capitate; clamp connections present.

\section{Gymnopilus earlei Murrill}

Distribution - São Paulo (Pegler 1997), Rio Grande do Sul (Cortez \& Coelho 2005), Paraná (De Meijer 2006, 2008 both as 'G. cf. earlei'), Mato Grosso do Sul (Bononi et al. 2008, as 'G. cf. earlei', Bononi et al. 2017).

Habitat - Growing in the roots of a fallen angiosperm trunk (e.g. Cortez \& Coelho 2005).

Note - According to Hesler (1969), this species is characterized by a large pileus up to 100 $\mathrm{mm}$ in diam., convex to plane, pale yellow to pale ferruginous, fibrillose to floccose-squamose; lamellae adnate to subdecurrent; veil arachnoid, yellow, fugacious; basidiospores 6-8.5 × 4-4.5 (5) $\mu \mathrm{m}$, ellipsoid in face-view and inequilateral in profile; pleurocystidia 23-28 $\times 5-8 \mu \mathrm{m}$, ventricose; cheilocystidia 18-25 $\times 3-7 \mu \mathrm{m}$, ventricose or conocyboid, apex subcapitate or noncapitate.

\section{Gymnopilus cf. flavidellus Murrill}

Distribution - Paraná (De Meijer 2006).

Habitat - On a chestnut stump in woods (Murrill 1917).

Note - According to Murrill (1917), G. flavidellus presents pileus 30-50 mm in diam., smooth, melleous to ochraceous; lamellae adnate or sinuate with decurrent tooth; veil arachnoid; basidiospores 8-9 × 5-6 $\mu \mathrm{m}$, 'ovoid'; cystidia not mentioned.

\section{Gymnopilus flavipunctatus (Speg.) Singer}

Distribution - São Paulo (Pegler 1997), Rio de Janeiro (Albuquerque et al. 2012).

Habitat - Growing on dead wood (Pegler 1997, Albuquerque et al. 2012).

Note - According to Albuquerque et al. (2012), the Brazilian record of this species presents pileus $22 \mathrm{~mm}$ in diam., fibrillose; lamellae decurrent; stipe squamulose, exannulate; basidiospores 7.7-9.5 $\times$ 4.8-5.3 $\mu \mathrm{m}$, ellipsoid; pleurocystidia and cheilocystidia not observed; clamp connections not informed.

\section{Gymnopilus hispidus (Massee) Murrill}

Distribution - São Paulo (Bononi et al. 1984).

Habitat - On rotten wood (Hesler 1969). 
Note - According to Hesler (1969), this species presents pileus up to $25 \mathrm{~mm}$ in diam., umbilicate to infundibuliform, ochraceous fulvous, squamulose, with erect squamules; lamellae subdecurrent; stipe fibrillose, veil or annulus not informed; basidiospores 6-8 $\times$ 4-4.5 $\mu \mathrm{m}$, ellipsoid in side view and slightly inequilateral in profile; pleurocystidia and cheilocystidia not seen; clamp connections present.

\section{Gymnopilus imperialis (Speg.) Singer}

Distribution - São Paulo (Pegler 1997), Paraná (De Meijer 2006).

Habitat - Growing on frondose trees (Pegler 1997).

Note - According to Hesler (1969), this species is characterized by the pileus up to $100 \mathrm{~mm}$ in diam., rufescent saffron to cinnamon saffron, golden ferruginous, golden fulvous, orange fulvous or fulvous, smooth when young then very densely and minutely fibrillose-squamulose with short and appressed fibrils; lamellae adnate or sinuate, with long decurrent tooth; annulus membranous; basidiospores $8-10 \times 7-8.5 \mu \mathrm{m}$, subglobose to broadly ellipsoid; pleurocystidia absent; cheilocystidia 30-40× 7-10 $\mu \mathrm{m}$, fusoid-ventricose, apex capitate or sub-capitate; clamp connections present.

\section{Gymnopilus marasmioides (Berk.) Singer}

Distribution - Amazonas (Berkeley \& Cooke 1877, as Agaricus marasmioides Berk., Pegler 1988, Singer 1965), São Paulo (Pegler 1997), Paraná (De Meijer 2006).

Habitat - Growing on dead wood (Pegler 1997).

Note - According to Singer (1965), this species presents pileus 5-30 mm in diam., subglabrous and minutely sulcate-striate all over when dry, fulvous, furfuraceous but glabrescent; lamellae adnate, perhaps sometimes emarginated or separate from the stipe; veil absent; basidiospores $6-9 \times 4.8-6.5 \mu \mathrm{m}(7-9 \times 5.5-6 \mu \mathrm{m}$ according to Pegler 1988); pleurocystidia to 33 $\mu \mathrm{m}$ long and $7 \mu \mathrm{m}$ wide, clavate and ventricose at upper and lower portion and constricted in the middle; cheilocystidia 21-23 × 5.5-6 $\mu \mathrm{m}$, mostly clavate, constricted bellow the subcapitate apex; clamp connections present.

\section{Gymnopilus pampeanus (Speg.) Singer}

Distribution - São Paulo (Pegler 1997); Rio Grande do Sul (Drechsler-Santos et al. 2007).

Habitat - Growing on Eucalyptus trunk (Pegler 1997).

Note - According to Pegler (1983), this species presents pileus 50-120 (-190) mm in diam., zinc orange to lemon chrome, surface minutely appressed fibrillose-squamulose; lamellae adnexed to sinuate adnate; annulus membranous, persistent; basidiospores 6.5-9.5 $\times 5-6.5 \mu \mathrm{m}$, subglobose to broadly ovoid; pleurocystidia absent or scattered and similar to cheilocystidia; cheilocystidia 23$32 \times 5-8 \mu \mathrm{m}$, fusoid to subventricose, apex sometimes subcapitate; clamp connections present.

\section{Gymnopilus panurensis (Berk.) Pegler}

Distribution - Amazonas (Berkeley 1856, Berkeley \& Cooke 1877, as Agaricus panurensis Berk., Pegler 1988).

Habitat - On the ground (Pegler 1988).

Note - According to Berkeley (1856) and Pegler (1988), this species has pileus 20-60 mm in diam., with triangular fulvous squamules; lamellae adnate; veil absent; basidiospores 8-10 × 5.5-7 $\mu \mathrm{m}(\mathrm{L}=9.5 \mu \mathrm{m} ; \mathrm{W}=6.3 \mu \mathrm{m} ; \mathrm{Qm}=1.52)$, broadly ovoid; pleurocystidia and cheilocystidia unknown.

\section{Gymnopilus peliolepis (Speg.) Singer}

Distribution - São Paulo (Spegazzini 1919, as Pholiota peliolepis, Singer \& Digilio 1952, Pegler 1997), Rio Grande do Sul (Singer 1953), Paraná (De Meijer 2006).

Habitat - On deciduous tree trunks (Hesler 1969). 
Note - According to Singer (1952), Hesler (1969), and Guzmán-Dávalos et al. (2008), this species presents pileus $20-70 \mathrm{~mm}$ in diam., red to deep purple squamules on pileus; lamellae sinuate-adnate to adnate-decurrent; veil cortinoid; basidiospores 6.8-9 $\times 4.3-5.8 \mu \mathrm{m}$ (Singer \& Digilio 1952), 6-8 (-9) × 4-4.5 $\mu \mathrm{m}$ (Hesler 1969), $6-8 \times 4.4-5.2 \mu \mathrm{m}$ from the spore print and 6.4$7.2 \times 4-4.8 \mu \mathrm{m}$ from the basidiomata (Guzmán-Dávalos et al. 2008), ellipsoid to oblong;

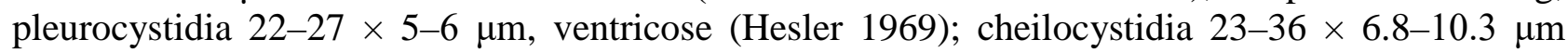
ampullaceous (Singer 1952) or 12-32 × 4-6 $\mu \mathrm{m}$, flask-shaped, ventricose to subfusoid, apex noncapitate or sometimes capitate (Hesler 1969); caulocystidia 33-73 $\times 5-12 \mu \mathrm{m}$, filamentous to subclavate (Hesler 1969); clamp connections present. Guzmán-Dávalos et al. (2008) pointed out that is difficult to have an adequate interpretation of $G$. peliolepis due to differences among several authors as observed above, as well as the presence of two classes of basidiospores, with different ornamentation, in the type specimen.

\section{Gymnopilus picreus (Pers.) P. Karst.}

Distribution - Rio Grande do Sul (Rick 1961)

Habitat - On conifer wood and sometimes on hardwood (Hesler 1969).

Note - According to Hesler (1969), this species presents pileus 10-20 (-50) mm in diam., subsquamulose, hygrophanous, rufous to bay brown cinnamon; lamellae adnate to decurrent; stipe exanulate; basidiospores 7-9(-9.5) $\times$ 4.5-5.5 (-6) $\mu \mathrm{m}$, ellipsoid or subovoid in face view, slightly inequilateral in profile; pleurocystidia 27-35 × 6-8 $\mu \mathrm{m}$, ventricose; cheilocystidia 23-33 $\times 4-7 \mu \mathrm{m}$, ventricose, apex capitate to non-capitate; caulocystidia 30-62 × 5-17 $\mu \mathrm{m}$, flask-shaped, apex capitate; clamp connections present.

\section{Gymnopilus pratensis Singer}

Distribution - São Paulo (Bononi et al. 1981).

Habitat - Growing on grass Singer \& Digilio (1952).

Note - According to Singer \& Digilio (1952), this species presents pileus $15 \mathrm{~mm}$ in diam., ferruginous ochraceous brown at centre then brown yellow at margin, minutely apressed fibrillose; lamellae adnate; stipe exannulate; basidiospores 6.8-7.5 $\times 5.5 \mu \mathrm{m}$; pleurocystidia absent; cheilocystidia 23-28 $\times 4.7-7 \mu \mathrm{m}$, cylindric at base to ventricose and with sharp and subcapitate apex with brownish resinous incrustations; clamp connections present.

\section{Gymnopilus psamminus (Berk.) Singer}

Distribution - Amazonas (Berkeley 1856, as Agaricus psamminus, Pegler 1988).

Habitat - On the ground (Pegler 1988).

Note - According to Pegler (1988), this species presents pileus $10 \mathrm{~mm}$ in diam., smooth, whitish; lamellae "attenuate behind and attached at top of stipe"; stipe exannulate; basidiospores 9.5-13 $\times 5-7 \mu \mathrm{m}(\mathrm{L}=11 \mu \mathrm{m}, \mathrm{W}=6 \mu \mathrm{m}, \mathrm{Qm}=1.80)$ elongate to subamigdaliform; pleurocystidia and cheilocystidia not mentioned; clamp connections not mentioned.

\section{Gymnopilus purpureosquamulosus Høil.}

Distribution - Paraíba (Magnago et al. 2013, as ‘G. purpureosquamulosus (Peck) Singer’).

Habitat - On decaying hardwood in mixed riparian forest, on living trunks of Phoenix canariensis near the sea, on a log in tropical forest, or on a wooden wall next to a palm (GuzmánDávalos et al. 2008).

Note - According to Guzmán-Dávalos et al. (2008), this species presents pileus (10-) 23-95 $\mathrm{mm}$ in diam., squamules lilac-pink, red fuchsia, reddish, rusty red, or pale brown when rain-soaked and purple when dry; lamella adnate, uncinate-adnate or shortly decurrent; with fugacious annulus in older stages; basidiospores (6.4-)7.2-9.6(-10.4) × (4-)4.7-6(-6.4) $\mu \mathrm{m}[\mathrm{Q}=1.33-1.80(-1.90)]$, ellipsoid to oblong then amygdaliform with obtuse to very few truncate apex; subhymenium cellular with some elongated elements; pleurocystidia absent or scarce; cheilocystidia 16-29(-40) $\times$ $3.2-8(-10) \mu \mathrm{m}$, lageniform or cylindrical with capitate to subcapitate apex; caulocystidia $22.4-73(-$ 
88) $\times 3.2-17 \mu \mathrm{m}$, cylindrical or clavate, few utriform or lageniform, apex obtuse, subcapitate or capitate; clamp connections present.

\section{Gymnopilus purpureogramminicola Silva-Junior \& Wartchow}

Distribution - Paraíba (Silva-Junior \& Wartchow 2015).

Habitat - Growing on grass (Silva-Junior \& Wartchow 2015).

Note - According to Silva-Junior \& Wartchow (2015), this species presents pileus 10-32 mm in diam., with purple, reddish brown or vinaceous squamules; lamellae short-decurrent; annulus fibrillose; basidiospores (6.1-)6.4-9.7(-10.2) $\times(4.1-) 4.6-6.7 \mu \mathrm{m}[\mathrm{L}=7.8 \mu \mathrm{m} ; \mathrm{W}=5.3 \mu \mathrm{m} ; \mathrm{Q}=$ (1.21-)1.23-1.74(-1.90), Qm = 1.45)], ellipsoid to few widely ellipsoid and elongate; subhymenium cellular; pleurocystidia and cheilocystidia not found; caulocystidia 25-32 × 5.5-6.5 $\mu \mathrm{m}$, slender clavate to lageniform; clamp connections present.

\section{Gymnopilus pyrrhus (Berk. \& M.A. Curtis) B.J. Rees}

Distribution - Paraná (De Meijer 2006).

Habitat - Growing on trunks (Dennis 1970).

Note - According to Dennis [1970, as Pyrroglossum pyrrhus (Berk. \& M.A. Curtis) Singer], the species has pileus to $25 \mathrm{~mm}$ in diam., light brown, smooth or tomentose; lamellae subdecurrent; stipe laterally attached, exannulate; basidiospores 5-6 $63.5 \mu \mathrm{m}$, shape not mentioned; pleurocystidia and cheilocystidia not mentioned.

\section{Gymnopilus russipes Hesler \& A.H. Sm.}

Distribution - Paraná (De Meijer 2008).

Habitat - On rotten fallen branch (Pegler 1983).

Note - According De Meijer (2008), it is characterized by the pileus $10-45 \mathrm{~mm}$ in diam., brown to brownish orange at centre then paler toward margin, smooth, dry, glabrous; lamellae adnexed to narrowly adnate; exannulate; basidiospores 8-9 × 6.5-7.5 $\mu \mathrm{m}$; pleurocystidia absent; cheilocystidia 18-23 × 4-7 $\mu \mathrm{m}$, lageniform-subtibiiform to tibiiform, apex slightly capitate.

\section{Gymnopilus spectabilis (Weinm.) A.H. Sm.}

Distribution - Rio Grande do Sul (Rick 1961)

Habitat - On conifer and deciduous logs, stumps, living and dead trunks or buried wood (Hesler 1969).

Note - According to Hesler (1969), this species possesses pileus (50-)80-180 mm in diam., buff-yellowor more rarely at first whitish, or "deep chrome”, "xanthine orange", "antimony yellow", to "sudan brown" or "raw sienna", nearly glabrous, sometimes appressed-fibrillose and minutely squamulose at centre; lamellae adnate to decurrent; membranous veil; basidiospores (7)8-10 $\times 4.5-5.5(-6) \mu \mathrm{m}$, ellipsoid to ovoid in side-view to slightly inequilateral in profile; pleurocystidia 21-33 × 6-7 $\mu \mathrm{m}$, ventricose, scattered; cheilocystidia 24-43 $\times 4-8 \mu \mathrm{m}$, ventricose, apex capitate or not capitate; caulocystidia (20-)36-65(-107) $\times(2.5-) 5-10 \mu \mathrm{m}$, somewhat similar to cheilocystidia; clamp connections present.

\section{Gymnopilus subtropicus Hesler}

Distribution - Paraná (De Meijer 2006), Paraíba (Magnago et al. 2015).

Habitat - On gum log (Hesler 1969).

Note - According to Hesler (1969) this species has pileus 10-45 mm in diam., yellowish, fibrillose; lamellae adnato-decurrent; stipe with arachnoid fugacious annulus; basidiospores (5)5.5-7 $\times 4-4.5 \mu \mathrm{m}$, ellipsoid in side-view and slightly inequilateral in profile; pleurocystidia 23-30 $\times 5-8 \mu \mathrm{m}$, ventricose; cheilocystidia 12-30 × 3-6 $\mu \mathrm{m}$, flask-shape to ventricose, apex non-capitate, more rarely capitate; caulocystidia 22-26 × 4-9 $\mu \mathrm{m}$, ventricose, cylindric or clavate at times capitate. 
Gymnopilus trailii (Berk. \& Cooke) Singer 1988).

Distribution - Amazonas (Berkeley \& Cooke 1877, as Agaricus trailli Berk. \& Cooke, Pegler

Habitat - On decayed wood (Pegler 1988).

Note - According to Berkeley \& Cooke (1877) and Pegler (1988), this species has pileus ' $1 / 2$ inch' (about $12 \mathrm{~mm}$ ) in diam., fulvous with erect squamules at surface; lamellae attachment not mentioned; membranous annulus; basidiospores 5.5-6 × 3.7-4.5 $\mu \mathrm{m}(\mathrm{L}=5.6 \mu \mathrm{m}, \mathrm{W}=4.2 \mu \mathrm{m}, \mathrm{Qm}$ $=1.30$ ), ovoid to short amygdaliform; pleurocystidia 18-25 $\times 5-7 \mu \mathrm{m}$, utriform, apex often subcapitate, numerous; cheilocystidia 24-30 × 5-7 $\mu \mathrm{m}$, sinuous-lageniform.

\section{Species with doubtful record}

Gymnopilus aureobrunneus (Berk. \& M.A. Curtis) Murrill 1990)

Distribution - Unknown place (Montagne 1856, as Cortinarius weddellianus Mont., Pegler

Habitat - On logs and stumps (Hesler 1969).

Note - According to Hesler (1969) this species has pileus 20-50 mm in diam., golden yellow, appressed-fibrillose, dry; lamellae adnate or adnexed; veil not reported; basidiospores 5.5-7.5 × 4$4.5 \mu \mathrm{m}$, ellipsoid in side-view and slightly inequilateral in profile; pleurocystidia absent; cheilocystidia 20-24 × 3-4 $\mu \mathrm{m}$, flask-shape, apex non-capitate. On the other hand, Pegler (1990) mentioned Cortinarius weddellianus with pileus 130-150 mm in diam., reddish yellow, slightly squamulose, and growing on soil. He suspected that this species could correspond to $G$. aureobrunneus.

\section{Final remarks}

From Brazil, 22 species of Gymnopilus are reported, occurring in the states of Amazonas, Mato Grosso do Sul, Paraíba, Paraná, Rio Grande do Sul, Santa Catarina, and São Paulo. However, some names cited here need to be reviewed to confirm their identity.

\section{Acknowledgements}

The authors wish to thank the support from the Brazilian agencies: 'Conselho Nacional de Desenvolvimento Científico e Tecnológico' $(\mathrm{CNPq})$ by funding the project 'Fungos agaricoides em áreas de Mata Atlântica e Caatinga no Estado da Paraíba’ (Edital Universal Proc. 420.448/2016-0) and providing 'Produtividade em Pesquisa' (Proc. 307922/2014-6 and Proc. 307947/2017-3) grant.

\section{References}

Albuquerque MP, De Carvalho-Júnior AA, Pereira AB, Putzke J. 2012 - Fungos da Reserva Biológica do Tinguá, Rio de Janeiro, Brasil: Espécies das famílias Entolomataceae, Physalacriaceae, Pluteaceae, Strophariaceae e Tricholomataceae. Caderno de Pesquisa Série Biologia 24, 83-108.

Anonymous. 2019 - Index Fungorum. International Mycological Association. http://www.indexfungorum.org/ (accessed 14 December 2019).

Berkeley MJ, Cooke MC. 1877 - The fungi of Brazil, including those collected by J.W.H. Trail, Esq. M.A., in 1874. Journal of the Linnean Society, Botany 15, 363-398.

Berkeley MJ. 1856 - Decades of Fungi LI-LIV: Rio Negro Fungi. Hooker Journal of Botany Kew Garden Miscelaneous 8, 129-144.

Bononi VLR, Mucci ESF, Yokomizo NKS, Guzmán G. 1984 - Agaricales (Basidiomycetes) do Parque Estadual de Campos do Jordão, SP, Brasil. Rickia 11, 85-89.

Bononi VLR, Oliveira AKM, Quevedo JR, Gugliotta AM. 2008 - Fungos macroscópicos do Pantanal do Rio Negro, Mato Grosso do Sul, Brasil. Hoehnea 35, 489-511. 
Bononi VLR, Trufen SFB, Grandi RAP. 1981 - Fungos macroscópicos do Parque Estadual das Fontes do Ipiranga, São Paulo, Brasil, depositados no Herbário do Instituto de Botânica de São Paulo. Rickia 9, 37-53.

Bononi VLR, Oliveira, AKM, Gugliotta, AMG, Quevedo, JR. 2017 - Agaricomycetes (Basidiomycota, Fungi) diversity in a protected area in the Maracaju Mountains, in the Brazilian central region. Hoehnea 44, 361-377.

Capelari M, Cortez VG, Neves MA, Baseia IG et al. 2015 - Agaricales. In: Lista de espécies da flora do Brasil. Jardim Botânico Do Rio De Janeiro. Disponível Em: $<$ http://floradobrasil.jbrj.gov.br/jabot/floradobrasil/FB95178>. Access 02 October 2019.

Cortez VG, Coelho G. 2005 - Additions to the mycobiota (Agaricales, Basidiomycetes) of Rio Grande Do Sul, Brazil. Iheringia, Série Botânica 60, 69-75.

De Meijer AAR. 2006 - Preliminary list of the macromycetes from the Brazilian state of Paraná. Boletim do Museu Botânico Municipal 68: 1-55.

De Meijer AAR. 2008 - Macrofungos notáveis das florestas de Pinheiro-do-Paraná. Embrapa Florestas, Colombo.

Dennis RWG. 1970 - Fungus flora of Venezuela and adjacent countries. Kew Bulletin Additional Series 3, 1-531.

Drechsler-Santos ER, Pastorini LH, Putzke J. 2007 - Primeiro relato de fungos Agaricales em fragmento de mata nativa em Frederico Westphalen - RS. Revista Brasileira de Biociências 5, 471-473.

Guzmán-Dávalos L, Mueller GM, Cifuentes J, Miller AN, Santerre A. 2003 - Traditional infrageneric classification of Gymnopilus is not supported by ribosomal DNA sequence data. Mycologia 95, 1204-1214.

Guzmán-Dávalos L, Contu M, Ortega A, Vizzini A et al. 2008 - New morphological and molecular data on Gymnopilus purpureosquamulosus and its phylogenetic relationships among similar species. Sydowia 60, 41-56

Hesler LR. 1969 - North American species of Gymnopilus. Mycologia Memoirs 3: 1-117.

Karstedt F, Stürmer SL. 2008 - Agaricales em áreas de floresta ombrófila densa e plantações de Pinus no estado de Santa Catarina, Brasil. Acta Botanica Brasilica 22, 1036-1043.

Kühner R. 1984 - Some mainlines of classification in the gill fungi. Mycologia 76, 1059-1074.

Magnago AC, Oliveira JJS, Furtado ANM, Urrea-Valencia S, Neves MA. 2013 - Mushroomscogumelos. In: Neves MA, Baseia IG, Drechsler-Santos ER, Góes-Neto A. Guide to the common fungi of the semiarid region of Brazil, TECC Editora, Florianópolis. Pp. 23-49.

Magnago AC, Furtado ANM, Urrea-Valencia S, Freitas AF, Neves MA. 2015 - New records of agaricoid fungi (Basidiomycota) from Paraíba, Brazil. Biotemas 28, 9-21.

Matheny PB, Moreau P-A, Vizzini A, Harrower E et al. 2015 - Crassisporium and Romagnesiella: two new genera of dark-spored Agaricales. Systematics and Biodiversity 13, 28-41.

Montagne JPFC. 1856 - Septième centurie de plantes cellulaires nouvelles, tant indigenes Qu' exottiques. Annales des Science Naturelles, Botanique Series 45, 333-374.

Murrill WA. 1917 - Agaricales, Agaricaceae (pars), Agariceae (pars). North American Flora 10, 145-226.

MycoBank Database. 2019 - Fungal Databases, Nomenclature \& Species Banks. Landcare Research, New Zealand. http://nzfungi.landcareresearch.co.nz (accessed 14 May 2013).

Pegler DN. 1983 - Agaric flora of Lesser Antilles. Kew Bulletin Additional Series 9, 1-668.

Pegler DN. 1988 - Agaricales of Brazil described by M.J. Berkeley. Kew Bulletin 43, 453-473.

Pegler DN. 1990 - Agaricales of Brazil described by J.P.F.C. Montagne. Kew Bulletin 45, 161177.

Pegler DN. 1997 - The agarics of São Paulo: An account of the agaricoid fungi (Holobasidiomycetes) of São Paulo State, Brazil. Royal Botanic Garden, Kew.

Putzke J. 1994 - Lista dos fungos Agaricales (Hymenomycetes, Basidiomycotina) referidos para Brasil. Caderno de Pesquisa Série Botânica 6, 3-186. 
Rick J. 1961 - Basidiomycetes Eubasidii in Rio Grande do Sul, Brasilia. Iheringia, Série Botânica 8, 296-450.

Robert V, Stegehuis G, Stalpers J. 2005 - The MycoBank engine and related databases. http://www.mycobank.org (accessed 14 December 2019).

Silva-Junior FCS, Wartchow F. 2015 - Gymnopilus purpureograminicola (Strophariaceae, Agaricomycetidae), a new species from Paraíba, Brasil. Nova Hedwigia 101, 395-402.

Singer R. 1953 - Type studies on Basidiomycetes. Lilloa 26, 57-159.

Singer R. 1965 - Interesting and new Agaricales from Brazil. Atas do Instituto de Micologia da Universidade do Recife 2, 15-59.

Singer R. 1986 - The Agaricales in modern taxonomy. Koeltz Scientific Books, Koenigstein.

Singer R, Digilio APL. 1952 ('1951') - Pódromo de la flora Agaricina Argentina. Lilloa 25: 5-461.

Spegazzini CL. 1919 - Reliquiae mycologicae tropicae. Boletín de la Academia Nacional de Ciencias en Córdoba 23, 365-609. 